MANCHESTER

1824

군 Economics

Discussion Paper Series

EDP-1708

\title{
Dynamic analysis of discontinuous best response with innovation
}

\author{
Fabio Lamantia \\ Mario Pezzino \\ Fabio Tramontana
}

February 2017

Economics

School of Social Sciences

The University of Manchester

Manchester M13 9PL 


\title{
Dynamic analysis of discontinuous best response with innovation
}

\author{
Fabio Lamantia \\ University of Calabria (Italy) and University of Manchester (UK) \\ fabio.lamantia@unical.it \\ Mario Pezzino \\ University of Manchester (UK) \\ mario.pezzino@manchester.ac.uk \\ Fabio Tramontana \\ Catholic University of the Sacred Heart, Milan (Italy) \\ fabio.tramontanal@unicatt.it
}

April 2017

\begin{abstract}
We study a Cournot duopoly where firms can decide to incur fixed costs in activities that improve their competitiveness (i.e. product development or process innovation). Innovation costs generate discontinuities in the firms' quantity best response functions and, in turn, a variety of equilibrium configurations, including multiple equilibria. We provide a dynamic global analysis of the equilibria and show the way in which firms' initial expectations regarding the rival's level of output are crucial in defining the configuration of the long run equilibrium.
\end{abstract}

Keywords: Process Innovation; Oligopolistic competition; Discontinuous best response functions; Global analysis; Learning.

JEL codes: C73; D21; D43.

\section{Introduction}

In many industries firms can increase their competitiveness investing in innovation to reduce costs (process innovation) or developing new products to better meet consumers' preferences and increase their willingness to pay (product innovation).

Several contributions in the literature have studied the problem of strategic investments in innovation. One very fruitful approach used to understand the 
effects of market and technical uncertainty as well as strategic interaction is the stream of research on real options (see e.g. [13]). Other contributions focus on innovation with multi-product competition and (production) capacity constraints (see [11], [12]). A different branch of literature explores the effects of various behavioral rules behind firms' decision making. Specifically, several scholars have questioned the assumption of players' perfect knowledge on the structure of the game. Some have thus formulated dynamic economic models based on learning effects, misperception of market elements and expectations on rivals' actions. One of the major contributors in this respect has been Carl Chiarella, who touched upon all these elements in a number of his works (see, among many others, [8], [9], [10], [7]).

Along the line of this last stream, our paper studies a dynamic Cournot duopoly with adaptive expectations learning, where firms can initially decide to invest in (product and/or process) innovation projects that will increase their competitiveness, before competing in the market selecting quantities. Innovation costs are assumed to be fixed. This is a sufficiently realistic assumption to describe those industries (e.g. pharmaceuticals and high-tech) experiencing large economies of scale and requiring significant sunk investments. In what follows, we focus on industries in which the fixed cost component (e.g. high-tech equipment, large pieces of machinery and advertising campaigns) are prevalent compared to variable costs (such as better raw materials or cheaper labour). The possibility of investing in product/process innovation that requires incurring fixed costs introduces interesting features to the well-known Cournot duopoly. In fact, it generates discontinuities in the firms' quantity best response functions and, for some parameter configurations, a multiplicity of stable Cournot-Nash equilibria, including asymmetric ones. This poses the necessity to introduce dynamic adjustments to solve equilibrium selection problems. In addition, and probably more importantly, we show that the likelihood that a particular equilibrium configuration may be reached in the long run depends on the setup of the learning process and on the initial expectations that each player forms regarding the output level of the rival. With this respect, we assume that players update their beliefs on rival's strategies according to adaptive expectations, which constitute a learning rule more sophisticated than static (or naive) expectations. As we point out in the paper, under this more realistic scheme, players can indeed learn to play symmetric as well as asymmetric Cournot-Nash equilibria. We also show that learning to play an equilibrium configuration is path-dependent. This calls for a full understanding of the dynamics of the system through its global properties. The importance of such global analysis in economics, and in particular in oligopoly models, has again been remarked several times also by Carl Chiarella (see for instance [1], [2]). Indeed, this kind of analysis has critical economic relevance. Firms may employ forms of strategic ambiguity and deliberate information leaks to the press in order to shape the expectations of rivals to their advantage. Similarly, industrial reforms (e.g. market deregulation) may induce firms to expect lower or higher levels of competition and create shocks to the dynamic path of the game, eventually, affecting the equilibrium selection. Moreover, the decision to participate/abandon trading agreements (e.g. the re- 
cent uncertainties connected to Brexit) may shape firms' expectations and have dramatic effects in terms of equilibrium selection in the long run.

Our model produces asymmetric equilibria where only one firm innovates. The innovator effectively dominates the market in equilibrium, earning higher profits and serving a larger portion of the market compared to the rival. As already pointed out in [14] in a static game, the existence of these asymmetric equilibria raises important antitrust issues. Indeed competition can produce a market configuration in which two ex-ante identical firms end up in an asymmetric equilibrium in which the market is dominated by one of them. However, [14] show that the equilibrium could also be produced if one of the firms decided to employ unlawful predatory strategies targeted at monopolizing the market. In other words in this environment predatory behavior may work as an equilibrium selection mechanism. The fact that the same asymmetric equilibria can be produced both by fair competition and predatory behavior makes the correct identification of antitrust cases problematic. ${ }^{1}$ Using only a static perspective, market data would be of little help, because they would describe a particular equilibrium, but they would not provide information about the way that particular equilibrium has been selected. We therefore study the local and global stability of each equilibrium, focusing on two particular cases. First, similar to [14], we consider the possibility that both firms can compete for the market incurring fixed costs to invest in product/process innovation (two-sided innovation). Second, we consider the possibility where only one firm can invest to boost competitiveness (one-sided innovation). This case is interesting because it allows us to study forms of natural product differentiation where, for example, the country of origin of a good may impose advantages/disadvantage on a brand (think of the importance of the "Made in" label for some brands).

The paper is organized as follows. Section two introduces the static model of two-sided innovation and describes the best response functions. Section three states the dynamic model and describes the different equilibria. Section four analyzes the main dynamic features of the system under naive and adaptive expectations. Section five proposes a variation of the previous model considering one-sided innovation. Section six concludes.

\section{The model}

Here we develop the static model of the game. We shall study its dynamic version in Section 3.

Let us assume that the market is served by two ex-ante identical firms. Firm $i, i=1,2$, produces quantity $x_{i}$. Both firms can decide to invest in product/process innovation and incur cost $k_{i}>0$. For the moment, let us focus out attention to the case in which $k_{1}=k_{2}=k$.

\footnotetext{
${ }^{1}$ The issue is known in the literature on predation in markets with winner-takes-all innovation. [16] explain that "predation is often indistinguishable from intense competition". See also [15] and [17].
} 
The inverse demand of a producer who does not innovate is

$$
p_{l}=a_{l}-b\left(x_{1}+x_{2}\right)
$$

whereas, by investing in innovation, the inverse demand is

$$
p_{h}=a_{h}-b\left(x_{1}+x_{2}\right)
$$

Parameters $a_{h} \geq a_{l}>0$ models the degree of vertical differentiation, see [18]. Firms face constant marginal costs. In particular the marginal cost is $c_{h}$ if the firm decides to invest in innovation and $c_{l}$ otherwise. Assume that $0<c_{h} \leq c_{l}$. Normalize $a_{l}-c_{l}=1$ and define $a \equiv a_{h}-c_{h}$. Let us assume that $a \in[1,2)$; $a \geq 1$ ensures that the investment $k$ can be seen both as process and product innovation that, given the linearity of the demand, would produce the result of an increase in the margin between the reservation price and marginal costs; $a<2$ ensures that in those asymmetric equilibria in which only one of the two firms decides to invest in innovation, the other firm still provides a positive quantity of output.

Player's $i$ earns profit

$\pi_{i}\left(x_{i}, x_{j}\right)=\left\{\begin{array}{l}\pi_{i, l}\left(x_{i}, x_{j}\right)=x_{i}\left(1-b\left(x_{i}+x_{j}\right)\right) \text { if firm } i \text { does not invest in innovation } \\ \pi_{i, h}\left(x_{i}, x_{j}\right)=x_{i}\left(a-b\left(x_{i}+x_{j}\right)\right)-k \text { if firm } i \text { invests in innovation }\end{array}\right.$

Given the production that player $i$ expects from player $j$, denoted by $x_{j}^{e}$, player $i$ solves the problem

$$
\arg \max _{x_{i}} \pi_{i}\left(x_{i}, x_{j}^{e}\right)
$$

If player $i$ does not invest in innovation, then the maximization of $\pi_{i, l}\left(x_{i}, x_{j}^{e}\right)$ with respect to $x_{i}$ and for a given level of $x_{j}^{e}$ (expected adversary's production) gives the 'best response' quantity $x_{i}^{*}=\frac{1}{2 b}-\frac{x_{j}^{e}}{2}$. Substituting back $x_{i}^{*}$ in $\pi_{i, l}\left(x_{i}, x_{j}^{e}\right)$ we get

$$
\begin{aligned}
\pi_{i, l}\left(x_{i}^{*}, x_{j}^{e}\right) & =x_{i}^{*}\left(1-b\left(x_{i}^{*}+x_{j}^{e}\right)\right)= \\
& =\frac{\left(1-b x_{j}^{e}\right)^{2}}{4 b}
\end{aligned}
$$

and analogously, in the case that player $i$ invests in innovation, it produces $x_{i}^{*}=\frac{a}{2 b}-\frac{x_{j}^{e}}{2}$ earning profits

$$
\pi_{i, h}\left(x_{i}^{*}, x_{j}^{e}\right)=\frac{\left(a-b x_{j}^{e}\right)^{2}}{4 b}-k
$$

Firm $i$ does not invest in innovation if condition $\pi_{i, l}\left(x_{i}^{*}, x_{j}^{e}\right)>\pi_{i, h}\left(x_{i}^{*}, x_{j}^{e}\right)$ holds. In other words, firm $i$ 's Best response can be written as follows: ${ }^{2}$

\footnotetext{
${ }^{2}$ The existence of discontinuities in the best response functions of the Cournot duopolists is not a special feature created by the assumption of linear demands. In what follows, to improve tractability, we limit ourselves to the case with linear demands. For related works with discontinuous best response functions see [25], [4], [6].
} 


$$
\begin{aligned}
x_{i}^{*} & =B\left(x_{j}^{e}\right)=\max \left[0,\left\{\begin{array}{c}
\frac{a}{2 b}-\frac{x_{j}^{e}}{2} \text { if } \pi_{i, l}\left(x_{i}^{*}, x_{j}^{e}\right) \leq \pi_{i, h}\left(x_{i}^{*}, x_{j}^{e}\right) \\
\frac{1}{2 b}-\frac{x_{j}^{e}}{2} \text { if } \pi_{i, l}\left(x_{i}^{*}, x_{j}^{e}\right)>\pi_{i, h}\left(x_{i}^{*}, x_{j}^{e}\right)
\end{array}\right]=\right. \\
& =\max \left[0,\left\{\begin{array}{l}
B_{H}\left(x_{j}^{e}\right)=\frac{a}{2 b}-\frac{x_{j}^{e}}{2} \text { if } 0 \leq x_{j}^{e} \leq \widehat{x} \\
B_{L}\left(x_{j}^{e}\right)=\frac{1}{2 b}-\frac{x_{j}^{e}}{2} \text { if } \widehat{x}<x_{j}^{e} \leq \frac{1}{b}
\end{array}\right]\right.
\end{aligned}
$$

where the break point is

$$
\widehat{x}=\frac{1+a}{2 b}-\frac{2 k}{a-1}
$$

Condition $x_{j}^{e} \leq \frac{1}{b}$ in (1) ensures non-negativity of production by firm $i$, i.e. $x_{i}^{*} \geq 0$. If in (2) it is $\widehat{x}<0$, then the best response (1) of firm $i$ reduces to the standard textbook case, with continuous best response:

$$
x_{i}^{*}=\widetilde{B}\left(x_{j}^{e}\right)=\max \left[0, \frac{1}{2 b}-\frac{x_{j}^{e}}{2}\right]
$$

Condition $\widehat{x}<0$ occurs for sufficiently high fixed cost of differentiation $k$, namely $k>\frac{a^{2}-1}{4 b}$. Thus, in the following we assume that $k \in\left[0, \frac{a^{2}-1}{4 b}\right]$, in order to rule out uninteresting cases.

If $\widehat{x} \geq \frac{1}{b}$ or equivalently $k \in\left[0, \frac{(a-1)^{2}}{4 b}\right]$, then the lower branch $B_{L}\left(x_{j}^{e}\right)$ in the best response (1) disappears. Stated differently, when the fixed cost to differentiate is sufficiently 'low', then it is always convenient to invest in innovation, given the expectation $x_{j}^{e}$. Therefore, firm $i$ 's best response when $k \in\left[0, \frac{(a-1)^{2}}{4 b}\right]$ simplifies to

$$
x_{i}^{*}=B\left(x_{j}^{e}\right)=\left\{\begin{array}{l}
\frac{a}{2 b}-\frac{x_{j}^{e}}{2} \text { if } 0 \leq x_{j}^{e} \leq \widehat{x} \\
0 \text { otherwise }
\end{array}\right.
$$

In what follows we shall focus our interest on values of $k \in\left(\frac{(a-1)^{2}}{4 b}, \frac{a^{2}-1}{4 b}\right)$ to ensure that both $B_{H}\left(x_{j}^{e}\right)$ and $B_{L}\left(x_{j}^{e}\right)$ in (1) lead to produce positive quantities $x_{i}^{*}$. Best response $B\left(x_{j}^{e}\right)$ in (3) has the well-known linear form with decreasing slope. Best response $\widetilde{B}\left(x_{j}^{e}\right)$ in (1) is a piecewise linear function with two branches both with slope $-\frac{1}{2}$ and a jump discontinuity at $\widehat{x}$, see Figure 1 .

\section{Oligopoly dynamics}

The previous Section described how best response functions are obtained for the duopoly model at hand. Here we discuss how firms dynamically update their production plans. As we shall see in this Section, this oligopoly game generates several coexisting equilibria, so the dynamic adjustment is essential to solve the equilibrium selection problem. 


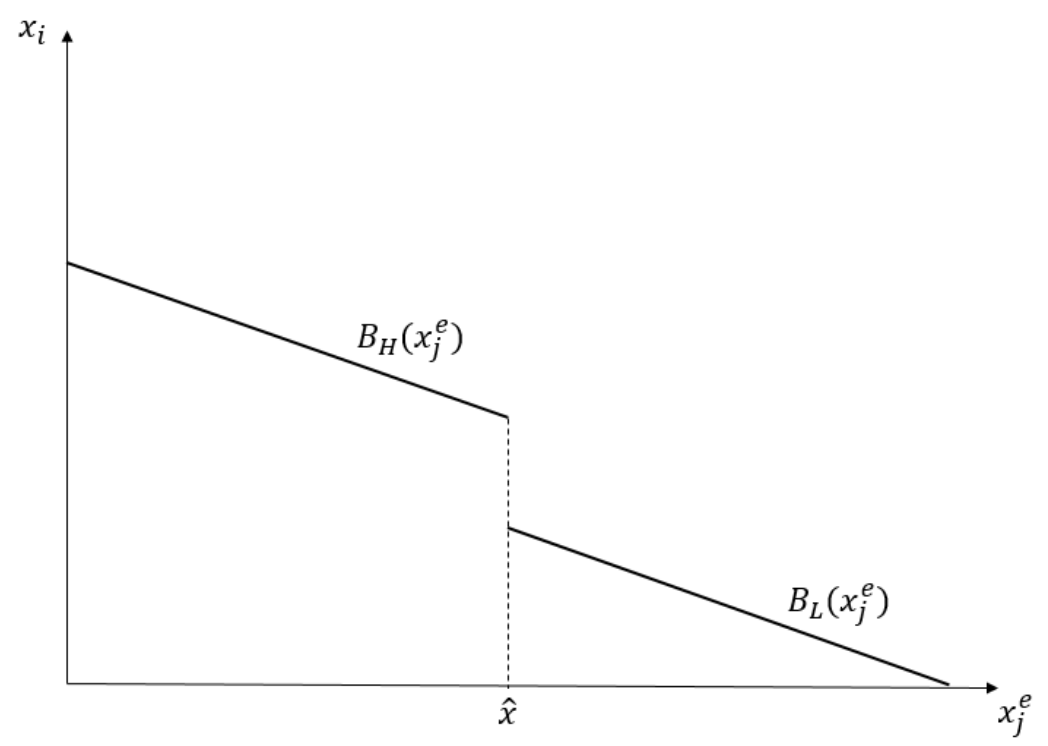

Figure 1: Best response function (1).

To employ a widely adopted framework, assume that firms update their beliefs according to adaptive expectations, ${ }^{3}$

$$
x_{i}^{e}(t+1)=x_{i}^{e}(t)+\alpha\left(x_{i}(t)-x_{i}^{e}(t)\right)
$$

In words, (5) says that the quantity that at time $t$ firm $j$ expects firm $i$ will produce at time $t+1$, denoted by $x_{i}^{e}(t+1)$, is given by firm's $j$ previous expectation, $x_{i}^{e}(t)$, corrected for the error observed between actual production by firm $i$ and firm's $j$ expected production for firm $i$ at time $t$. With this respect, parameter $\alpha \in[0,1]$ represents agents' propensity to revisit expectations or the speed of adjustment of the system. In this paper, we assume the same speed of adjustment for both players. Note that for $\alpha=0$, firm $j$ does not revise its expectations about the rival's production, i.e. $x_{i}^{e}(t+1)=x_{i}^{e}(t)$, whereas for $\alpha=1$, firms have naive (or Cournot) expectations, i.e. firms assume that the best proxy for their rival's next-period production is the current production, i.e. $x_{i}^{e}(t+1)=x_{i}(t)$. Coupling the equation of adaptive expectations (5) with best response functions $B($.$) , see (1), a dynamical oligopoly game with adaptive$ expectations is defined by iterating the map $P: \mathbb{R}^{4} \rightarrow \mathbb{R}^{4}$ given by:

$$
P:\left\{\begin{array}{l}
x_{j}(t+1)=B\left(x_{i}^{e}(t+1)\right) \\
x_{i}^{e}(t+1)=x_{i}^{e}(t)+\alpha\left(x_{i}(t)-x_{i}^{e}(t)\right)
\end{array} \quad i, j=1,2 ; i \neq j\right.
$$

\footnotetext{
${ }^{3}$ Empirical evidences that adaptive expectations are consistent with the mechanism of individuals' expectation formation are reported in [20] and [21]. As explained in [2], dynamics under adaptive expectations is equivalent to partial adjustment towards the best response with naive expectations.
} 
The state variables of model (6) are expected productions, $x_{1}^{e}(t), x_{2}^{e}(t)$, and actual productions $x_{1}(t), x_{2}(t)$, for the two players, hence $P$ is a fourdimensional map. Interestingly, as already highlighted in [19] and [3], the dynamics of the map $P$ can be completely captured by a lower dimensional map depending on expectations only. In fact, by substituting the first equation in (6) written at period $t$ for agent $i$, namely $x_{i}(t)=B\left(x_{j}^{e}(t)\right)$, into the second equation in (6) one obtains

$$
T:\left\{x_{i}^{e}(t+1)=x_{i}^{e}(t)+\alpha\left(B\left(x_{j}^{e}(t)\right)-x_{i}^{e}(t)\right), i, j=1,2 ; i \neq j\right.
$$

which is a two-dimensional discontinuous map in the space of expected quantities $x_{1}^{e}(t), x_{2}^{e}(t)$, i.e. $T: \mathbb{R}^{2} \rightarrow \mathbb{R}^{2}$. In this way, it is possible to study the dynamic properties of model (6) through model (7): map $T$ generates the dynamics of expectations, which then, determine the dynamics of productions through the transformation from the beliefs space to production space through the first equation of (6). Observe that at equilibrium expected quantities coincide with actual quantities. Also attractors (e.g. cycles or more complex attractors) of map $P$ can be obtained through the simpler model $T$, as explained in the following. Also the stability properties remain unchanged by $T$, since all time-t actual productions can be obtained through the (non-dynamical and) simultaneous transformations $x_{j}(t)=B\left(x_{i}^{e}(t)\right), i, j=1,2 ; i \neq j$.

To easy the notation, in the following we write $x_{i}^{e}(t)=x_{i}$ and $x_{i}^{e}(t+1)=x_{i}^{\prime}$. Written extensively, map $T$ assumes the following form

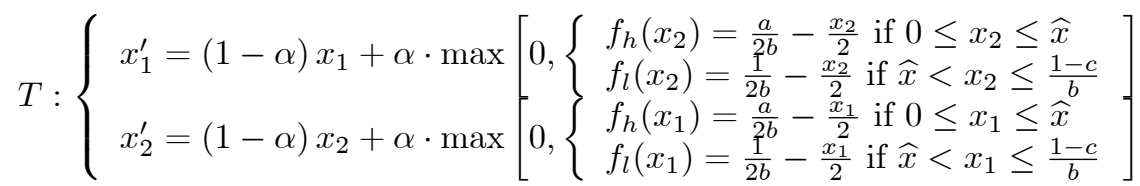

where $\widehat{x}$ is given in (2).

Next we study the existence and stability of the equilibria of the map (8) whose state variables are the beliefs $x_{i}^{e}(t)$. Importantly, any equilibrium in the belief space is also a Cournot-Nash equilibria in the space of the produced quantities. Notice that, although the best response functions are linear, the discontinuity at $\widehat{x}$ entails that no symmetric equilibria or multiple equilibria could exist for (8). We sum up the main results on existence and stability of equilibria in the following proposition.

Proposition 1 Consider map $T$ in (8) and define

$$
\begin{array}{ll}
\bar{k}_{h}=\frac{(a-1)(3+a)}{12 b} & \bar{k}_{l}=\frac{(a-1)(1+3 a)}{12 b} \\
\bar{k}_{h l}^{-}=\frac{(a-1)(5-a)}{12 b} & \bar{k}_{h l}^{+}=\frac{(a-1)(5 a-1)}{12 b}
\end{array}
$$

with

$$
\bar{k}_{h l}^{-}<\bar{k}_{h}<\bar{k}_{l}<\bar{k}_{h l}^{+}
$$


1. If $0<k<\bar{k}_{h l}^{-}$, then there exists a unique equilibrium

$$
E_{h h}=\left(\frac{a}{3 b}, \frac{a}{3 b}\right)
$$

where both firms invest in innovation. Moreover, $E_{h h}$ is locally asymptotically stable;

2. If $\bar{k}_{h l}^{-}<k<\bar{k}_{h}$ then, in addition to $E_{h h}$, there exist two additional equilibria

$$
E_{h l}=\left(x_{h}^{*}, x_{l}^{*}\right)=\left(\frac{2 a-1}{3 b}, \frac{2-a}{3 b}\right)
$$

and

$$
E_{l h}=\left(x_{l}^{*}, x_{h}^{*}\right)
$$

where $x_{h}^{*}$ denotes the quantity by the innovator and $x_{l}^{*}$ the quantity by the non-innovator. $E_{h h}, E_{h l}$ and $E_{l h}$ are locally asymptotically stable;

3. If $\bar{k}_{h}<k<\bar{k}_{l}$ then only $E_{l h}$ and $E_{h l}$ exist and are both locally asymptotically stable;

4. If $\bar{k}_{l}<k<\bar{k}_{h l}^{+}$then, in addition to $E_{l h}$ and $E_{h l}$, there exists an additional equilibrium

$$
E_{l l}=\left(\frac{1}{3 b}, \frac{1}{3 b}\right)
$$

where both firms are non-innovators. $E_{l l}, E_{h l}$ and $E_{l h}$ are locally asymptotically stable;

5. If $k>\bar{k}_{h l}^{+}$than only $E_{l l}$ exists and is locally asymptotically stable.

Proof See the Appendix.

Not surprisingly, the firm that invests in innovation dominates the market and produces more than the rival, being $x_{h}^{*}>x_{l}^{*}$. Denoting by $\pi_{m p}^{*}$ the equilibrium profit for a firm playing $m \in\{l, h\}$ when the rival plays $p \in\{l, h\}$, notice that $\pi_{h l}^{*} \geq \pi_{h h}^{*} \geq \pi_{l l}^{*} \geq \pi_{l h}^{*}$. Being the only innovator in the market is clearly the most advantageous equilibrium for a firm: the innovator dominates the market and earns higher profits. On the other hand, being the non-innovator when the rival decides to innovate is the worst possible outcome for the non-innovator. Thus, the presence of innovation fixed costs and the resulting discontinuities in the best response functions produce equilibrium configurations in which one of the two firms dominates the market, even if ex-ante the two firms are assumed to be identical. Such asymmetric equilibrium has been described here as the result of fair competition, but it could also be produced by unlawful predatory behavior (as explained in [14]). This raises significant antitrust difficulties in recognizing whether market dominance as described in equilibria $E_{h l}$ and $E_{l h}$ is the product of fair or unlawful competition. 
The presence of multiple stable equilibria motivates a global analysis of the dynamics of the system, in order to identify which trajectories converge to a particular equilibrium or if some trajectories converge to an attractor different from an equilibrium. For this reason, we present in the following an analysis of the possible attractors of the system and their basins of attraction. This analysis is also relevant from an economic point of view, since it can reveal whether an asymmetric equilibrium is the product of fair competition or the result of unlawful practices that have taken the system to a different dynamic path.

\section{Two-sided innovation}

Here we analyze the dynamics of the model under symmetric innovation costs for the two firms. Initially we provide analytical results on the attractors and the respective basins of attraction for the particular case of naive expectations. This constitutes an important benchmark often considered in the oligopoly literature and sheds light on general key insights of the model. We then describe the model with general adaptive expectations through numerical analysis.

\subsection{Naive Expectations}

Let us consider here as a benchmark case, the scenario in which both firms may react to the best response with naive expectation, whose dynamics is obtained through (8) with speed of adjustment $\alpha=1$. This benchmark case will prove to be very insightful, while also being analytically tractable. In addition, notice that equilibria configuration and stability are not affected by the particular value of parameter $\alpha$. As we shall discuss later, variations in $\alpha$ will only have important effects on the shape of the basins of attraction.

Let us denote by $\widetilde{T}$ map (8) with $\alpha=1$. Map $\widetilde{T}$ is characterized by a second iterate with separate variables. As proved in [5], this kind of maps usually has the peculiarity of coexistence of several stable equilibria. Moreover the dynamic behavior of such a duopoly model can be fully characterized by the one dimensional map

$$
X^{\prime}=F(X)=f_{m}\left(f_{p}(X)\right), m, p \in\{l, h\}
$$

that governs our model.

If we include trajectories leading to the Milnor attractor $X=0$ (corresponding to $O(0,0)$ for the two-dimensional map $\widetilde{T}$ ), which we will analyze separately in the following, map $F$ is in general discontinuous and can be formed by pieces 
of five different maps:

$$
\begin{array}{llll}
f_{h h}(X)=f_{h} \circ f_{h}(X)=\frac{a}{4 b}+\frac{X}{4} & \text { if } 0 \leq X \leq \widehat{x} & \text { and } & 0 \leq f_{h}(X) \leq \widehat{x} \\
f_{l h}(X)=f_{l} \circ f_{h}(X)=\frac{2-a}{4 b}+\frac{X}{4} & \text { if } 0 \leq X \leq \widehat{x} & \text { and } & \widehat{x}<f_{h}(X) \leq \frac{1}{b} \\
f_{h l}(X)=f_{h} \circ f_{l}(X)=\frac{2 a-1}{4 b}+\frac{X}{4} & \text { if } \widehat{x}<X \leq \frac{1}{b} & \text { and } & 0 \leq f_{l}(X) \leq \widehat{x} \\
f_{l l}(X)=f_{l} \circ f_{l}(X)=\frac{1}{4 b}+\frac{X}{4} & \text { if } \widehat{x}<X \leq \frac{1}{b} & \text { and } & \widehat{x}<f_{l}(X) \leq \frac{1}{b} \\
f_{0}(X)=0 & \text { otherwise } &
\end{array}
$$

The fixed points corresponding to the four (non-constant) linear maps are:

$$
\begin{aligned}
& f_{h h} \Longrightarrow X_{h h}^{*}=\frac{a}{3 b} \\
& f_{l h} \Longrightarrow X_{l h}^{*}=\frac{2-a}{3 b} \\
& f_{h l} \Longrightarrow X_{h l}^{*}=\frac{2 a-1}{3 b} \\
& f_{l l} \Longrightarrow X_{l l}^{*}=\frac{1}{3 b}
\end{aligned}
$$

and they are locally stable, as the slopes of the linear maps are all equal to $\frac{1}{4}$. It must be noticed that, given that the map $F$ is in general discontinuous, the local stability of each existing fixed point does not imply its global stability despite the linearity of the single maps.

So the questions concerning existence and local stability of the fixed points are not two different questions but are the same. Taking that into consideration, we only need the find the conditions on the parameters that permit to the fixed point to be inside to the domain of the corresponding map. Such conditions are summarized in the following Proposition:

\section{Proposition 2}

Consider map F in (14) and (15) and the innovation costs thresholds in (9).

1. If $0<k<\bar{k}_{h l}^{-}$then $X_{h h}^{*}$ exists and is a locally stable fixed point for the map $F$;

2. If $\bar{k}_{h l}^{-}<k<\bar{k}_{h}$ then $X_{h h}^{*}, X_{l h}^{*}$ and $X_{h l}^{*}$ exist and are locally stable fixed points for the map $F$;

3. If $\bar{k}_{h}<k<\bar{k}_{l}$ then $X_{l h}^{*}$ and $X_{h l}^{*}$ exist and are both locally stable fixed points for the map $F$;

4. If $\bar{k}_{l}<k<\bar{k}_{h l}^{+}$then $X_{l l}^{*}, X_{l h}^{*}$ and $X_{h l}^{*}$ exist and are locally stable fixed points for the map $F$; 
5. If $k>\bar{k}_{h l}^{+}$then $X_{l l}^{*}$ exists and is a locally stable fixed point for the map $F$.

[5] show that there exists a correspondence between the fixed points of the one-dimensional map $F$ and corresponding periodic points of the two-dimensional map $\widetilde{T}$. In particular, given the symmetry between the reaction curves of the two firms, we have that to each fixed point of $F$ it corresponds a periodic cycle on the diagonal of the phase plane of $\widetilde{T}$, that is a symmetric period point. In particular, the fixed points $X_{l l}^{*}$ and $X_{h h}^{*}$ correspond to the symmetric equilibria $E_{l l}$ and $E_{h h}$, respectively. Instead, the always coexisting fixed points $X_{l h}^{*}$ and $X_{h l}^{*}$ correspond to the points of a symmetric 2-cycle for the map $\widetilde{T}$, that is $C_{1}\left(x_{h}^{*}, x_{h}^{*}\right)$ and $C_{2}\left(x_{l}^{*}, x_{l}^{*}\right)$.

Besides that, from [5] we also know that when $N$ fixed points $X_{1}^{*}, X_{2}^{*}, \ldots, X_{N}^{*}$ (with $X_{1}^{*}<X_{2}^{*}<\ldots<X_{N}^{*}$ ) characterize the one-dimensional map $F$, several coexisting attractors characterize $\widetilde{T}$, whose points fill the Cartesian product $\left\{X_{1}^{*}\right\} \times\left\{X_{N}^{*}\right\}$.

So, from the Proposition 2, we must take into consideration five different scenarios:

\subsubsection{Scenario 1: Both the firms innovate}

This scenario occurs for the lowest values of the differentiation costs:

$$
0<k<\bar{k}_{h l}^{-}
$$

In this case $X_{h h}^{*}$ is the only fixed point of $F$, to which corresponds the equilibrium $E_{h h}$ for the two-dimensional map $\widetilde{T}$. All the feasible trajectories converge to this fixed point, where both firms innovate.

The following figures are obtained by keeping fixed the parameters $a=1.3$, $b=1$, whence $\bar{k}_{h l}^{-}=0.0925$. Figure 2 is obtained with $k=0.018$, so that Scenario 1 holds. In Figure 2a we can see the one-dimensional map $F$ whose piece $f_{h h}$ determines the fixed point $X_{h h}^{*}$. In Figure $2 \mathrm{~b}$ we have the corresponding reaction curves for the duopolists, where we can see the intersection in $E_{h h}$. Finally, Figure 2c shows that all initial beliefs generating feasible trajectories indeed converge to $E_{h h}$, that is the Cournot-Nash equilibrium in the actual production space where both firms innovate.

\subsubsection{Scenario 2: Multistability with at least one innovating firm}

When $\bar{k}_{h l}^{-}<k<\bar{k}_{h}$ the one-dimensional map $F$ has three fixed points. For the twodimensional map $\widetilde{T}$, these coexisting fixed points on the branches $f_{h h}, f_{h l}$ and $f_{l h}$ correspond to the fixed point $E_{h h}$ (both firms innovate) and to a symmetric two-cycles made up by the points $C_{1}^{l}\left(x_{l}^{*}, x_{l}^{*}\right)$ and $C_{1}^{h}\left(x_{h}^{*}, x_{h}^{*}\right)$ (firms cyclically innovate and do not innovate). However, they are not the only coexisting attractors for the map $\widetilde{T}$. The Cartesian product $\left\{x_{l}^{*}\right\} \times\left\{x_{h}^{*}\right\}$ is filled with two asymmetric equilibria $E_{l h}$ and $E_{h l}$ (only one firm innovate) and two cycles of 

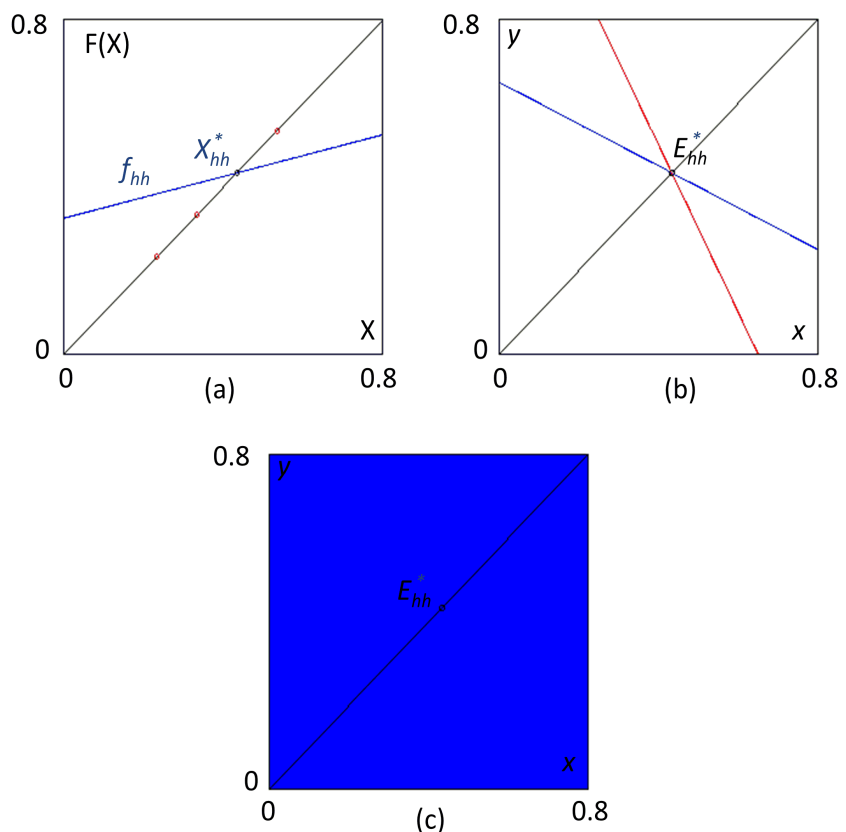

Figure 2: Two-sided innovation - Scenario 1. $a=1.3, b=1, k=0.018$. (a) Map $F$ and its unique fixed point $X_{h h}^{*}$. (b) Reaction curves for the duopolists whose intersection represents the unique Cournot-Nash equilibrium $E_{h h}$. (c) Basin of attraction of $E_{h h}$ (blue). 

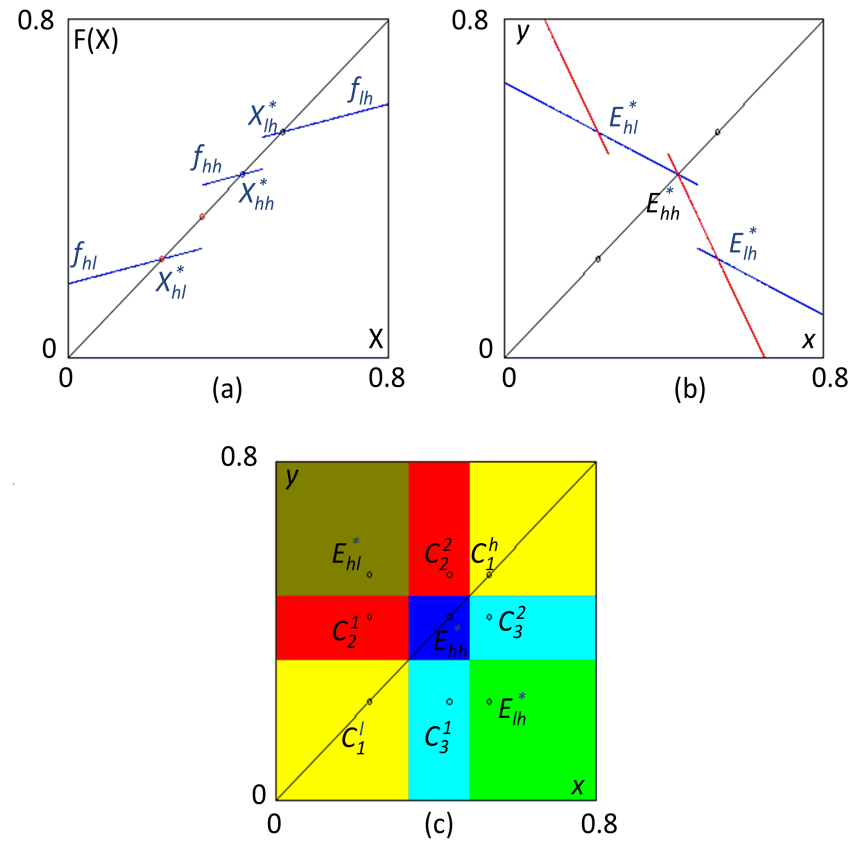

Figure 3: Two-sided innovation - Scenario 2. $a=1.3, b=1, k=0.1$. (a) Map $F$ and its fixed points $X_{h l}^{*}, X_{h h}^{*}$ and $X_{l h}^{*}$. (b) Reaction curves for the duopolists whose intersection represents the coexisting Cournot-Nash equilibria $E_{h l}, E_{h h}$ and $E_{l h}$ (c) Basins of attraction of: $E_{h l}$ (dark yellow), $E_{h h}$ (blue), $E_{l h}$ (green), symmetric 2-cycle $\left\{C_{1}^{l}, C_{1}^{h}\right\}$ (yellow), asymmetric 2-cycle $\left\{C_{2}^{1}, C_{2}^{2}\right\}$ (red) and asymmetric 2-cycle $\left\{C_{3}^{1}, C_{3}^{2}\right\}$ (cyan).

period-2 with points $C_{2}^{1}\left(x_{l}^{*}, \frac{a}{3 b}\right)$ and $C_{2}^{2}\left(\frac{a}{3 b}, x_{h}^{*}\right)$ for one cycle and $C_{3}^{1}\left(\frac{a}{3 b}, x_{l}^{*}\right)$ and $C_{3}^{2}\left(x_{h}^{*}, \frac{a}{3 b}\right)$ for the symmetric one.

This case is depicted in Figure 3. Considering that in our example it is $\bar{k}_{h}=0.1075$, we fixed $k=0.1$ in Figure 3 to obtain an example of scenario 2 . Figure $3 \mathrm{a}$ shows the three fixed points of $F$, whereas Figure $3 \mathrm{~b}$ and $3 \mathrm{c}$ show the reaction curves and the basins of attraction of the coexisting cycles, respectively. Moreover, the borders of the basins of attraction of the coexisting attractors are not related to the presence of saddle cycles, that do not exist in our case, but are related to the discontinuities of the map $F(X)$. In fact, the borders of the basins are marked by the discontinuity points of $F(X)$, where from the domain of $f_{h l}$ we pass to the domain of $f_{h h}$ and where from it we move to the domain of $f_{l h}$ (see [26]). This is clearly seen by inspecting Figures 3a and 3c. Moreover, the symmetry properties of the map are responsible for the symmetric rectangular shapes of the basins (see for details also [5]). The same mechanisms still work in the next scenarios as well. 

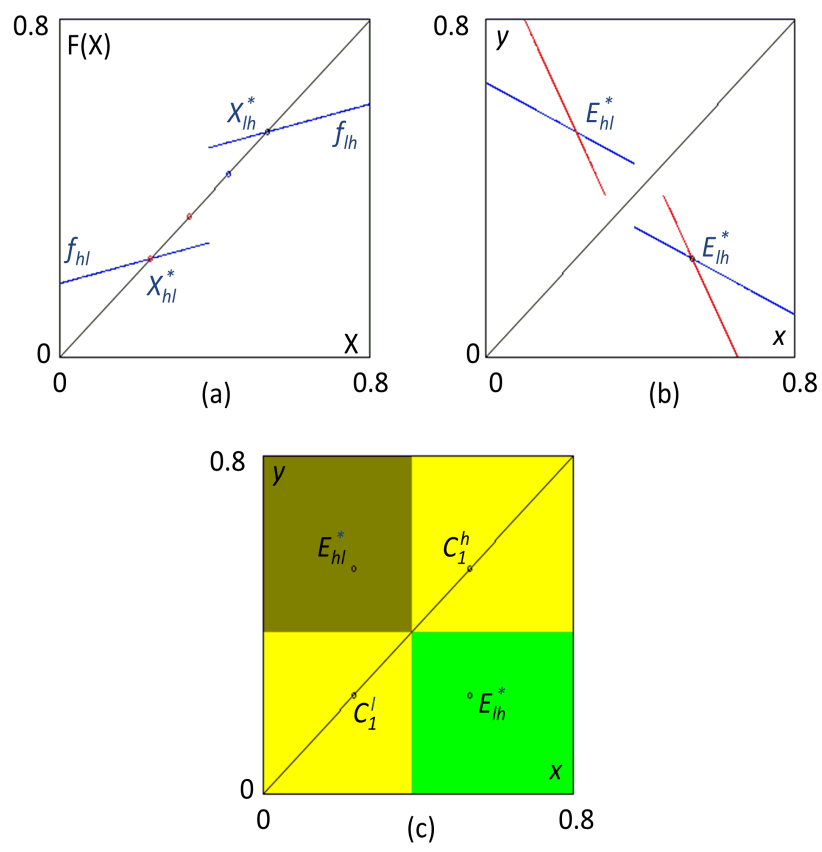

Figure 4: Two-sided innovation - Scenario 3. $a=1.3, b=1, k=0.115$. (a) Map $F$ and its fixed points $X_{h l}^{*}$ and $X_{l h}^{*}$. (b) Reaction curves for the duopolists whose intersection represents the coexisting asymmetric Cournot-Nash equilibria $E_{h l}$ and $E_{l h}$ (c) Basins of attraction of: $E_{h l}$ (dark yellow), $E_{l h}$ (green) and symmetric 2-cycle $\left\{C_{1}^{l}, C_{1}^{h}\right\}$ (yellow).

\subsubsection{Scenario 3: Asymmetric innovation equilibria and cycles}

When the cost of differentiation is higher than $\bar{k}_{h}$ but still lower than $\bar{k}_{l}$ we are in a new scenario. $F$ only admits two fixed points $X_{l h}^{*}$ and $X_{h l}^{*}$ that correspond to the symmetric 2 -cycle made up by $C_{1}^{l}\left(x_{l}^{*}, x_{l}^{*}\right)$ and $C_{1}^{h}\left(x_{h}^{*}, x_{h}^{*}\right)$ for the map $\widetilde{T}$. Without any other fixed point of $F(X)$, The Cartesian product is filled with the two asymmetric equilibria $E_{l h}$ and $E_{h l}$.

In our example $\bar{k}_{l}=0.1225$, so we fixed $k=0.115$ in Figure 4 to be in this scenario. Figure 4a shows the two fixed points of $F$. Reaction curves and basins of attraction are depicted in Figures $4 \mathrm{~b}$ and $4 \mathrm{c}$.

\subsubsection{Scenario 4: Multistability with at least one non-innovating firm}

When $\bar{k}_{l}<k<\bar{k}_{h l}^{+}$the one-dimensional map $F$ has again three fixed points but, besides $X_{l h}^{*}$ and $X_{h l}^{*}$ now the branch $f_{l l}$ intersects the bisector originating the fixed point $E_{l l}$. As a consequence this scenario looks like Scenario 2, with the 

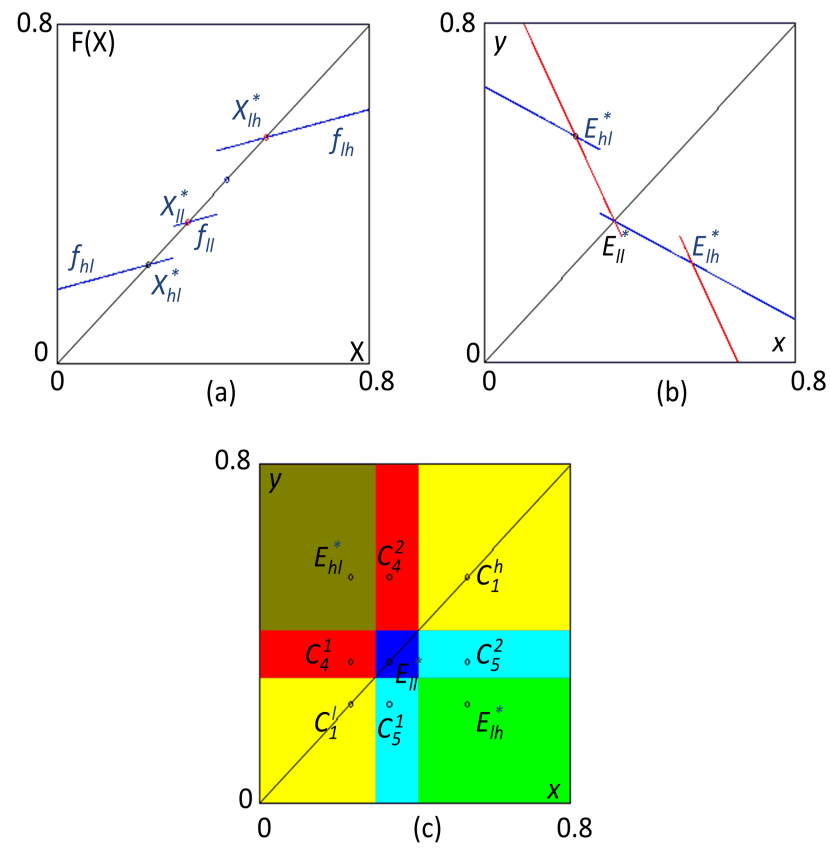

Figure 5: Two-sided innovation - Scenario 4. $a=1.3, b=1, k=0.128$. (a) Map $F$ and its fixed points $X_{h l}^{*}, X_{l l}^{*}$ and $X_{l h}^{*}$. (b) Reaction curves for the duopolists whose intersection represents the coexisting Cournot-Nash equilibria $E_{h l}, E_{l l}$ and $E_{l h}$ (c) Basins of attraction of: $E_{h l}$ (dark yellow), $E_{l l}$ (blue), $E_{l h}$ (green), symmetric 2-cycle $\left\{C_{1}^{l}, C_{1}^{h}\right\}$ (yellow), asymmetric 2-cycle $\left\{C_{4}^{1}, C_{4}^{2}\right\}$ (red) and asymmetric 2-cycle $\left\{C_{5}^{1}, C_{5}^{2}\right\}$ (cyan).

differences due to the existence of $E_{l l}$ instead of $E_{h h}$. So, besides $E_{l h}$ and $E_{h l}$, the map $\widetilde{T}$ is characterized by the coexistence of three 2-cycles: the first one is the symmetric $C_{1}^{l}\left(x_{l}^{*}, x_{l}^{*}\right)$ and $C_{1}^{h}\left(x_{h}^{*}, x_{h}^{*}\right)$, the second has periodic points $C_{4}^{1}\left(x_{l}^{*}, \frac{1}{3 b}\right)$ and $C_{4}^{2}\left(\frac{1}{3 b}, x_{h}^{*}\right)$ and the third is made up by $C_{5}^{1}\left(\frac{1}{3 b}, x_{l}^{*}\right)$ and $C_{5}^{2}\left(x_{h}^{*}, \frac{1}{3 b}\right)$. With our parametrization, it is $\bar{k}_{h l}^{+}=0.1375$ and we fixed in Figure $5 k=0.128$ to be in this scenario.

\subsubsection{Scenario 5: Both firms do not innovate}

In the final scenario, for the highest values of the differentiation cost (i.e. $\left.k>\bar{k}_{h l}^{+}\right)$the Cournot-Nash equilibrium is the one without differentiation $E_{l l}^{*}$, corresponding to the unique steady state of the one-dimensional map $X_{l l}^{*}$. In Figure 6 we used $k=0.176$ and all the feasible trajectories converge to $E_{l l}^{*}$. 


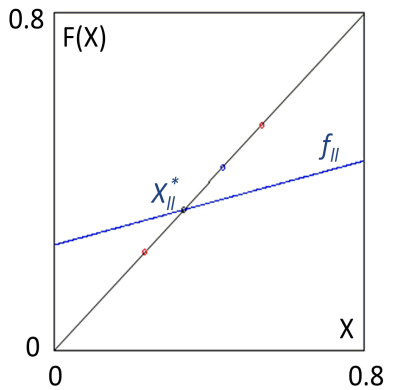

(a)

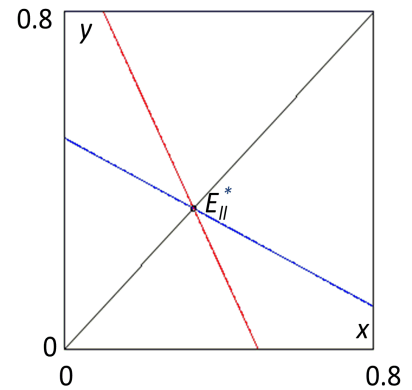

(b)

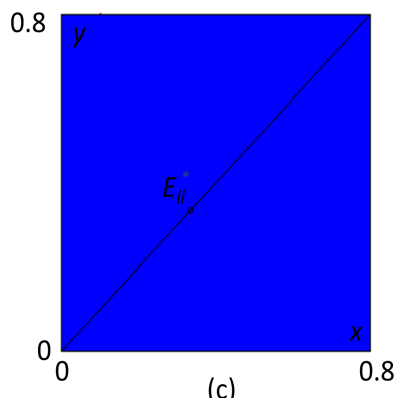

(c)

Figure 6: Two-sided innovation - Scenario 5. $a=1.3, b=1, k=0.176$. (a) Map $F$ and its unique fixed point $X_{l l}^{*}$. (b) Reaction curves for the duopolists whose intersection represents the unique Cournot-Nash equilibrium $E_{l l}$. (c) Basin of attraction of $E_{l l}$ (blue). 

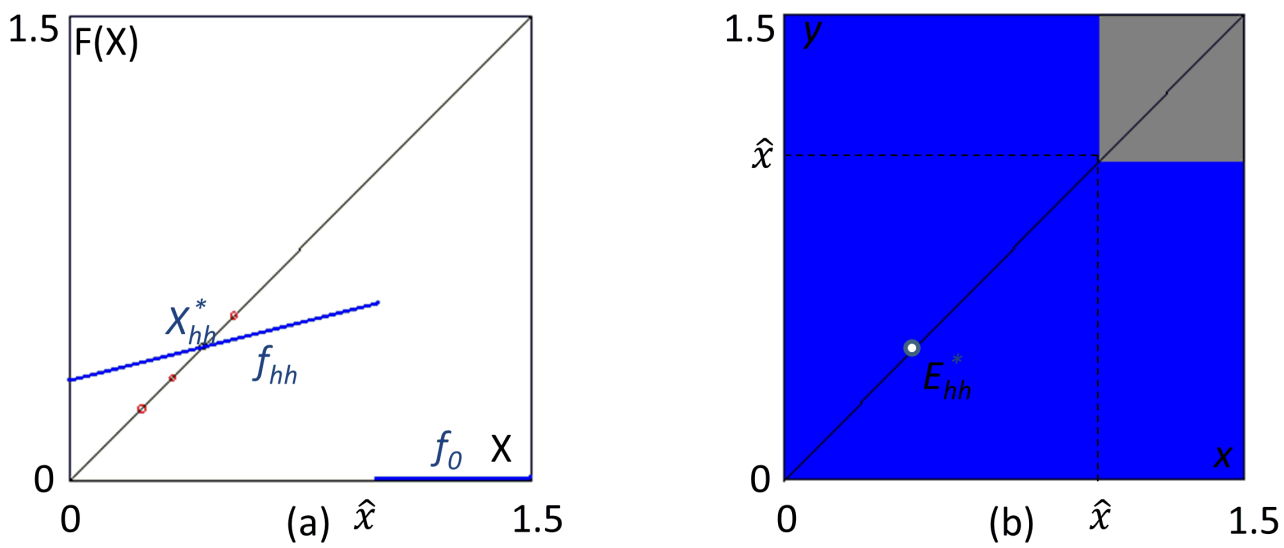

Figure 7: (a) Map $F$ with branch $f_{0}$ mapping points to the origin $(0,0)$. (b) Zomming out of Figure $2 c$ showing the basin of equilibrium $E_{h h}$ (blue) and the basin of the origin (gray), e.g. initial beliefs leading to unfeasible trajectories.

\subsubsection{The Milnor attractor}

As we have already mentioned, although not stable in Lyapunov sense the origin $O(0,0)$ is a stable attractor in Milnor (or weak) sense (see [22]) for the twodimensional map $\widetilde{T}$ even if it is not locally stable. In fact, the origin still has a basin of attraction with positive Lebesgue measure. This is only apparently a contradiction and is due to the existence of zero-branches in the definition of the map $F(X)$ in (14) and (15), avoiding unfeasible (negative) values of the production. This may occur for particularly high values of $X$, over a certain threshold $\widetilde{X}$. An example of its occurrence is depicted in Figure 7a, obtained zooming out Figure 2a. For $0 \leq X \leq \widehat{x}$, function $f_{l l}$ applies, while for $X>\widehat{x}$ we are in the domain of $f_{0}$, so every point is mapped to the origin. For the twodimensional map $T$ it means that in the plane $(x, y)$ any initial condition such that $x>\widehat{x}$ (or $y>\widehat{x}$ for symmetry reasons) belongs to the basin of attraction of the Milnor attractor at the origin, as can be seen in Figure $7 \mathrm{~b}$, zoom out of Figure 2c.

\subsection{Adaptive expectations}

Up to now, we have addressed the model of two-sided innovation under naive expectations. This benchmark case allowed us to provide analytical results on the existence of fixed points and cycles of period two. When a stable cycle of period two emerges, the long run play converges to a couple of beliefs, and hence of productions, oscillating between the two values of the cycle. As these expec- 
tations are systematically wrong, what we observe is that players are unable to learn the dynamics of the system. Of course, this is a rather implausible result produced by the restrictive and extreme assumption of 'rational' players with naive expectations (see [5] for a discussion on this issue).

We should instead expect rational players to learn from past mistakes, recognize cyclic trajectories and revise their expectations accordingly. Moving away from naive expectations, in this section we employ adaptive expectations as described in equation (5) with $\alpha<1$, allowing players to gradually learn about the opponent's strategies.

The stability of cycles of period two is detailed in the following proposition

Proposition 3 Consider map $T$ in (8). If a 2-cycle for (8) exists, then it is locally asymptotically stable.

Proof See the Appendix.

We show numerically that for smaller values of $\alpha$, even if stable cycles of period two continue to exist, their basins of attraction shrink to negligible size whereas the set of initial beliefs leading to the Cournot-Nash equilibria increases. For $\alpha$ sufficiently small, those cycles disappear.

Let us concentrate our discussion on an interesting scenario such as number 4 , where equilibria involve at least one non-innovating firm (the discussion of the other scenarios would be qualitatively similar and here omitted). The presence of 2-cycles with naive expectations $(\alpha=1)$ is due to the fact that players do not learn about the cycle and expect the rival to maintain a constant level of outcome. Figure 8a reproposes the example already discussed in Figure 5c.

A reduction of $\alpha$ entails that the new expected quantity at each time period is anchored to the old expectation and updated according to the error in the old expectation through equation (5). For the two-dimensional map $T$ in (8), this implies a deformation of the boundaries of all the basins of attraction. In fact, map $T$ is no more characterized by a second iterate with separable variables and the image of an horizontal (resp. vertical) line is not a vertical (resp. horizontal) line anymore. So the borders of the basins of attractions now are not horizontal and vertical lines, even if they are still related with the discontinuities of the map. Nevertheless, all the attractors survive for $\alpha$ sufficiently high, see Figure $8 \mathrm{~b}$, where $\alpha=0.9$.

The symmetric 2-cycle $\left\{C_{1}^{l}\left(x_{l}^{*}, x_{l}^{*}\right), C_{1}^{h}\left(x_{h}^{*}, x_{h}^{*}\right)\right\}$ in Figure $8 \mathrm{~b}$ is very close to touch the boundary of its basin of attraction, represented in yellow. A slight change of the speed of adjustment $\alpha$ can result in the disappearance of the 2-cycle together with its basin of attraction. This is an example of a contact bifurcation (see [23]), due to contacts between an attractor and the boundary of its basin, which determines the disappearance of the attractor, the 2-cycle in this case, as shown in Figure 8c.

Further reductions of $\alpha$ produce similar effects on the basins of attraction. In particular, the points of the asymmetric 2-cycles $\left\{C_{4}^{1}\left(x_{l}^{*}, \frac{1}{3 b}\right), C_{4}^{2}\left(\frac{1}{3 b}, x_{h}^{*}\right)\right\}$ and $\left\{C_{5}^{1}\left(\frac{1}{3 b}, x_{l}^{*}\right), C_{5}^{2}\left(x_{h}^{*}, \frac{1}{3 b}\right)\right\}$ get closer and their basins, depicted as the red and cyan regions respectively, shrink in size, see Figure 8d,e,f,g. For $\alpha$ sufficiently 
small, e.g. $\alpha=0.2$ in Figure $8 \mathrm{~h}$, any initial trajectory is attracted to a Nash equilibrium of the game, be it the symmetric one $E_{l l}$ or an asymmetric one.

\section{One-sided innovation}

In this Section, we consider the possibility that only one firm, say, firm 1 can decide to invest $k_{1}$ and earn a margin equal to $a .^{4}$ Firm 2 instead faces an insurmountable cost $k_{2}$. This special case is particularly realistic and interesting. In addition to the standard interpretation where only one of the two firms has the know-how to innovate and obtain a margin equal to $a$, this case provides also a framework to study an industry where competition between firms is influenced by some form of natural product differentiation. If the brands and products of the two firms are differentiated in the eyes of consumers by some unique, exogenously given characteristic, then one of the players may have a competitive advantage over the rival. For example, due to differences in missions and historical positioning of the brands, only one of the competitors can be in a position to exploit the advantage of incurring a fixed cost (e.g. charity donation or green environmental investment) and being recognized (and rewarded) by the market as a socially or environmentally concerned firm. The fixed cost incurred by firms can be indeed interpreted as the cost of advertising campaigns that firms need to incur in order to persuade consumers of their social or environmental responsibility. For some firms it is more expensive (sometimes impossible) than for others to convince consumers of operating according to motives other than profit maximization or market dominance. The case of natural product differentiation is also particularly important in an international context. Firms competing in an international market need to face and understand the competitive (dis-)advantage that can be created by the country of origin of the brand. This implies that there may be room for governments to invest in national labels or consumer education in order to increase or eliminate the competitive advantage created by product differentiation. It is therefore not surprising that firms and Governments may invest in "Made In" labels. Depending on the particular industry, country labels such as Made in Italy, Made in Germany and Made in Japan may bear indeed very different messages for consumers. The asymmetric case considered in this subsection provides room for interesting policy considerations. For example, when should we expect a Government to subsidize innovation (e.g. lowering $k$ ) or invest in national labels (with the effect of lowering $k$ or even increasing $a$ ) to take advantage of natural product differentiation? The question is particularly important when at least one of the two firms is not domestic. This implies that the policymakers' approach to the problem will significantly depend on the nationality of the firms serving the market. For example if both firms are foreign, it is reasonable to assume that a policymaker will assess the role of innovation/product differentiation based on the effect on consumer surplus. If instead one of the firms is domestic, the Government could

\footnotetext{
${ }^{4}$ The case in which only firm 2 will be able to invest in process/product innovation is analogous and thus disregarded here.
} 

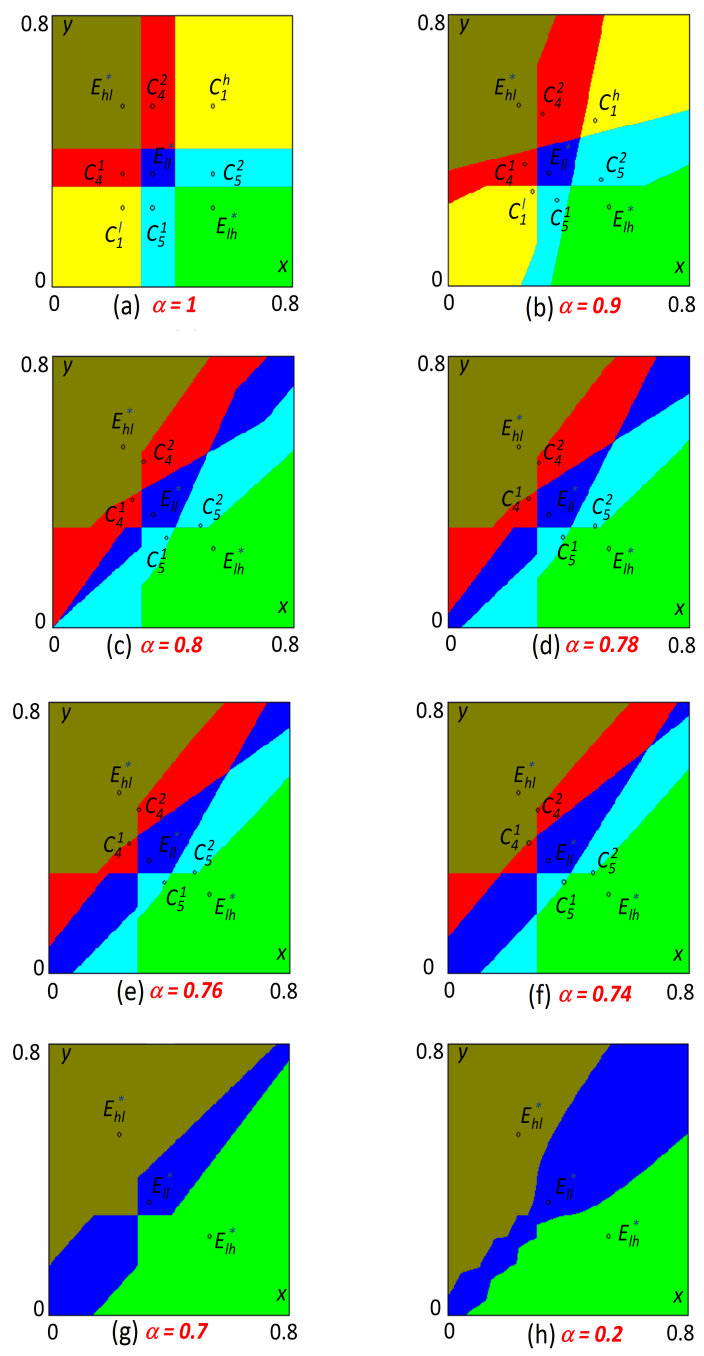

Figure 8: Two-sided innovation - Adaptive expectations. Basins of attraction of $E_{h l}$ (dark yellow), $E_{l l}$ (blue), $E_{l h}$ (green), symmetric 2-cycle $\left\{C_{1}^{l}, C_{1}^{h}\right\}$ (yellow), asymmetric 2-cycle $\left\{C_{4}^{1}, C_{4}^{2}\right\}$ (red), asymmetric 2-cycle $\left\{C_{5}^{1}, C_{5}^{2}\right\}$ (cyan). Parameters as in Figure 5 for decreasing values of the speed of adjustment $\alpha$. 
subsidize the innovation of the domestic firm or invest in media campaigns to boost the domestic label against the foreign one. In what follows we shall continue focusing on the case of innovation, but similar conclusions could be drawn for the case of natural product differentiation. We show that the multiplicity of equilibria survives in this case, so that a dynamic analysis of the problem is again essential.

Let us start the analysis with asymmetric players by considering the case in which $k_{2}>\frac{\left(a^{2}-1\right)}{4 b}>k_{1}$. As previously explained, this implies that firm 2 will never innovate, bearing too high costs for doing that. Therefore, firm 2 employs the standard best response (3), whereas firm 1 uses (1) or (4), depending, re-

spectively, whether $k_{1} \in\left(\frac{(a-1)^{2}}{4 b}, \frac{\left(a^{2}-1\right)}{4 b}\right)$ or $k_{1} \in\left[0, \frac{(a-1)^{2}}{4 b}\right]$. The analogous of map $T$ in (8) with only firm 1 innovating becomes

$$
T_{1}:\left\{\begin{array}{l}
x_{1}^{\prime}=(1-\alpha) x_{1}+\alpha \cdot \max \left[0,\left\{\begin{array}{c}
f_{h}\left(x_{2}\right)=\frac{a}{2 b}-\frac{x_{2}}{2} \text { if } 0 \leq x_{2} \leq \widehat{x} \\
f_{l}\left(x_{2}\right)=\frac{1}{2 b}-\frac{x_{2}}{2} \text { if } \widehat{x}<x_{2} \leq \frac{1}{b}
\end{array}\right]\right. \\
x_{2}^{\prime}=(1-\alpha) x_{2}+\alpha \cdot \max \left[0, f_{l}\left(x_{1}\right)=\frac{1}{2 b}-\frac{x_{1}}{2}\right]
\end{array}\right.
$$

where $\widehat{x}$ is given in (2) with $k=k_{1}$. Reasoning in a similar way as for the symmetric case, we can show that the following equilibria exist.

\section{Proposition 4}

Consider map $T_{1}$ in (17) with $k=k_{1}$ and $\bar{k}_{l}, \bar{k}_{h l}^{+}$in (9).

- If $0<k_{1}<\bar{k}_{l}$ then the only fixed point of (17) is $E_{h l}=\left(x_{h}^{*}, x_{l}^{*}\right)$ in (11).

- If $\bar{k}_{l}<k_{1}<\bar{k}_{h l}^{+}$then (17) admits two fixed points, given by $E_{h l}$ and $E_{l l}=\left(x_{l}^{*}, x_{l}^{*}\right)$ in $(13)$

- If $\bar{k}_{h l}^{+}<k_{1}$ then the only fixed point of (17) is $E_{l l}$.

- Any equilibrium of (17) is always locally asymptotically stable.

Proof. The proof is analogous to the case with two-sided innovation and therefore omitted.

Next we discuss in detail the case of one-sided innovation with naive expectations.

\section{$5.1 \quad$ Naive expectations}

In the case of naive expectations, the dynamics is given by the map $T_{1}$ in (17) with $\alpha=1$ and denoted by $\widehat{T}$ in the following. Also map $\widehat{T}$ has a second iterate with separate variables, so it can be studied through the one-dimensional map $G$, defined as

$$
X_{1}^{\prime}=G\left(X_{1}\right)=f\left(g\left(X_{1}\right)\right)
$$


This map is in general discontinuous and made up by two pieces: ${ }^{5}$

$$
\begin{array}{ll}
f_{h l}\left(X_{1}\right)=f_{h} \circ f_{l}\left(X_{1}\right)=\frac{2 a-1}{4 b}+\frac{X_{1}}{4} & \text { if } 0 \leq f_{l}\left(X_{1}\right) \leq \widehat{x} \\
f_{l l}\left(X_{1}\right)=f_{l} \circ f_{l}\left(X_{1}\right)=\frac{1}{4 b}+\frac{X_{1}}{4} & \text { if } \widehat{x}<f_{l}\left(X_{1}\right) \leq \frac{1}{b}
\end{array}
$$

characterized by two fixed points: $X_{h l}^{*}$ for $f_{h l}\left(X_{1}\right)$ and $X_{l l}^{*}$ for $f_{l l}\left(X_{1}\right)$, see (16). These fixed points are always locally stable whenever they exist. Similarly to the two-sided innovation case, we have the following result:

\section{Proposition 5}

Consider map $G$ in (18) and (19) and the innovation costs thresholds $\bar{k}_{l}$ and $\bar{k}_{h l}^{+}$in $(9)$.

- If $0<k_{1}<\bar{k}_{l}$ then $X_{h l}^{*}$ exists and is a locally stable fixed point for the map $G$;

- If $\bar{k}_{l}<k_{1}<\bar{k}_{h l}^{+}$then $X_{h l}^{*}$ and $X_{l l}^{*}$ exist and are locally stable fixed points for the map $G$;

- If $\bar{k}_{h l}^{+}<k_{1}$ then $X_{l l}^{*}$ exists and is a locally stable fixed point for the map $G$.

The correspondence between the dynamic properties of the one-dimensional map $G$ and those of the two-dimensional map $\widehat{T}$ is similar to the previous case, but now the two firms are not symmetric (i.e. only one may innovate); each fixed point of $G$ corresponds to a fixed point of the map $\widehat{T}$ according to:

$$
x_{1}^{*}=X^{*} \text { and } x_{2}^{*}=f\left(x_{1}^{*}\right)
$$

When there is only one fixed point for $G$ then there is only one fixed point for $\widehat{T}$. Instead, when two fixed points of $G$ coexist then the two-dimensional map $\widehat{T}$ has three locally stable attractors: two fixed points and a cycle of period two, whose points fill the Cartesian product in the two-dimensional plane.

Summarizing, we identify three scenarios:

\subsubsection{Scenario 1: Asymmetric innovation equilibrium}

This scenario occurs for the lowest values of the innovation costs:

$$
0<k_{1}<\bar{k}_{l}
$$

In this case $X_{h l}^{*}$ is the only fixed point of $G$, to which corresponds the fixed point $E_{h l}=\left(X_{h l}^{*}, f\left(X_{h l}^{*}\right)\right)$ for the two-dimensional map $\widehat{T}$. All the feasible trajectories converge to $E_{h l}$.

\footnotetext{
${ }^{5}$ Here for simplicity we disregard the piece $f_{0}=0$, as the considerations related to this branch are similar to the two-sided cases.
} 

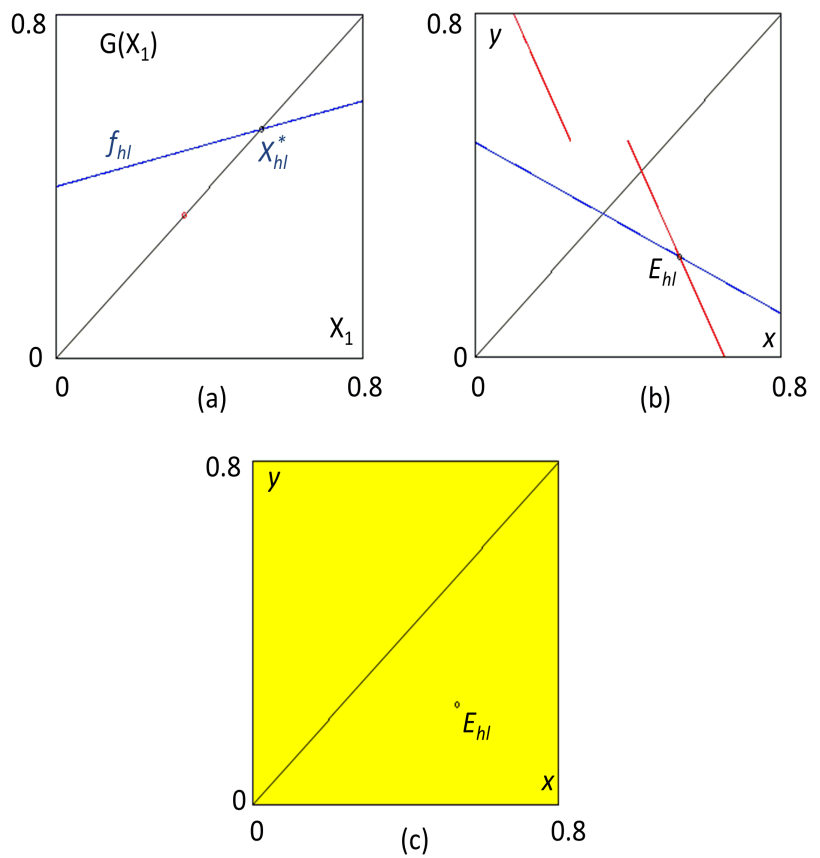

Figure 9: One-sided innovation - Scenario 1. $a=1.3, b=1, k_{1}=0.097$. (a) Map $G$ and its fixed point $X_{h l}^{*}$. (b) Reaction curves for the duopolists whose intersection represents the asymmetric Cournot-Nash equilibrium $E_{h l}$ (c) Basins of attraction of $E_{h l}$ (yellow).

Keeping fixed the parameters $a=1.3$ and $b=1$ as in our leading example with two-sided innovation, it is $\bar{k}_{l}=0.1225$. Figure 9 is obtained with $k_{1}=$ 0.097, ensuring that we are in Scenario 1. In Figure 9a we can see the onedimensional map $G$ whose piece $f_{l l}$ determines the fixed point $X_{h l}^{*}$. In Figure $9 \mathrm{~b}$ we have the corresponding reaction curves of the duopolists, where we can see the intersection at $E_{h l}$. Finally, Figure $9 \mathrm{c}$ shows that all the feasible trajectories converge to $E_{h l}$.

\subsubsection{Scenario 2: Multistability of Cournot-Nash equilibria}

When $\bar{k}_{l}<k_{1}<\bar{k}_{h l}^{+}$the one-dimensional map $G$ has two fixed points. Map $\widehat{T}$ admits the two fixed points $E_{h l}$ and $E_{l l}$ and the 2-cycle that closes the Cartesian product, whose points are $C^{1}\left(x_{l}^{*}, f\left(x_{h l}^{*}\right)\right)$ and $C^{2}\left(x_{h l}^{*}, x_{l}^{*}\right)$. Being $\bar{k}_{h l}^{+}=0.1375$, in Figure 10 we fixed $k_{1}=0.131$ to be in scenario 2. In Figure 10a we can see the two fixed point of the one-dimensional map $G$, and in Figure 10b the corresponding best response functions showing the Cournot-Nash equilibria for the two-dimensional map $\widehat{T}$. In Figure 10c the basins of attraction of the three 

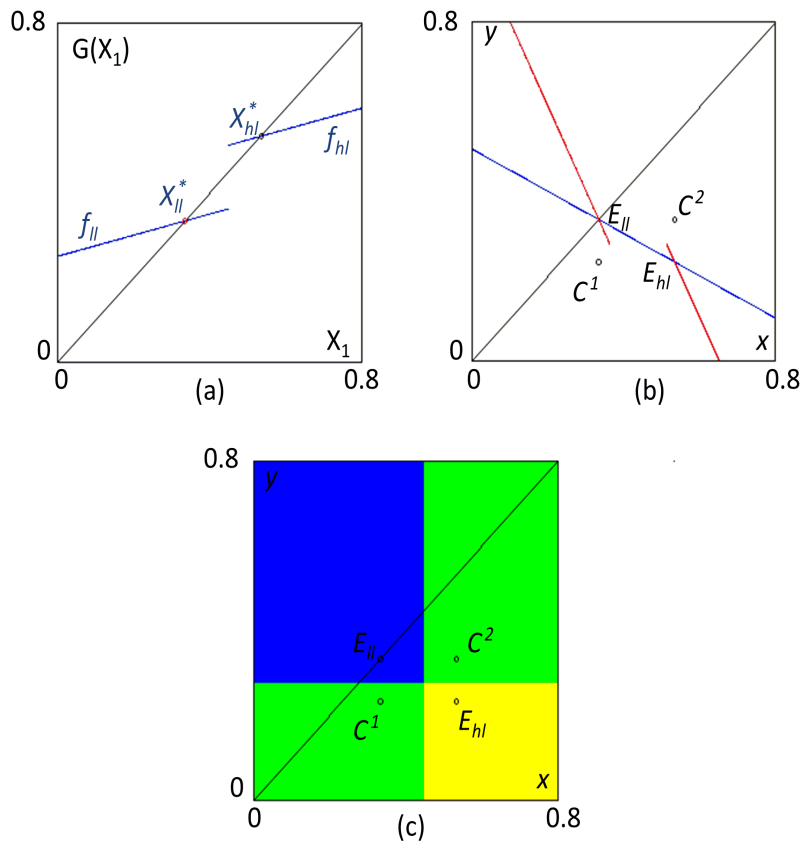

Figure 10: One-sided innovation - Scenario 2. $a=1.3, b=1, k_{1}=$ 0.131. (a) Map $G$ and its fixed points $X_{h l}^{*}$ and $X_{l l}^{*}$ (b) Reaction curves for the duopolists whose intersections represent Cournot-Nash equilibrium $E_{h l}$ and $E_{l l}$ (c) Basins of attraction of: $E_{h l}$ (yellow) $E_{l l}$ (blue) and 2-cycle $\left\{C^{1}\left(x_{l}^{*}, f\left(x_{h l}^{*}\right)\right), C^{2}\left(x_{h l}^{*}, x_{l}^{*}\right)\right\}$ (green).

coexisting attractors are represented in different colors.

\subsubsection{Scenario 3: Symmetric equilibrium without innovation}

For high values of $k_{1}$ (i.e. $k_{1}>\bar{k}_{h l}^{+}$) we have again a scenario with only one fixed point, named $X_{l l}^{*}$, for $G$ to which it corresponds the fixed point $E_{l l}$ for the two dimensional map $\widehat{T}$, with coordinates: $E_{l l}\left(X_{l l}^{*}, f\left(X_{l l}^{*}\right)\right)$. Figures 11a,b,c are obtained with $k_{1}=0.155$.

\subsection{Adaptive expectations}

Here we briefly develop an numerical exploration of the asymmetric case along the same line of Section 4.2. We only focus the discussion on scenario 2, which was the most interesting due to multistability of attractors.

Figure 12a depicts again the example of Figure 10c with naive expectations $(\alpha=1)$. Reducing $\alpha$ has the similar effect discussed before. The set of initial 


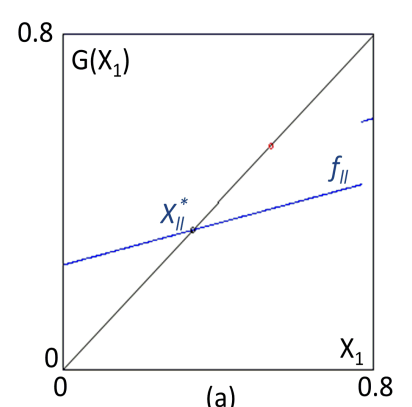

(a)

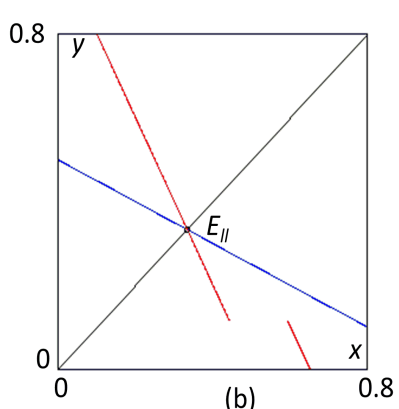

(b)

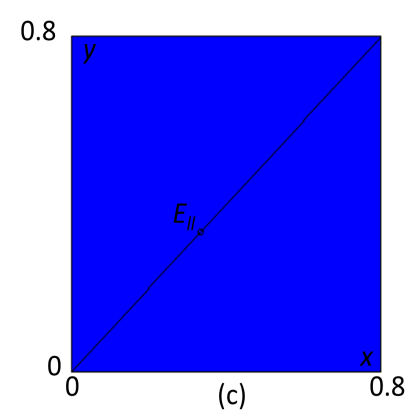

Figure 11: One-sided innovation - Scenario 3. $a=1.3, b=1, k_{1}=0.155$. (a) Map $G$ and its fixed point $X_{l l}^{*}$ (b) Reaction curves for the duopolists whose intersection represents the Cournot-Nash equilibrium $E_{l l}$ (c) Basins of attraction of $E_{l l}$ (blue). 
beliefs leading to convergence to a Cournot-Nash equilibrium increases, see Figure 12b,c. Again, learning is path-dependent. For $\alpha$ sufficiently low, the basins of attraction of the 2-cycle shrink to the point of becoming negligible, see Figure 12 d,e. Eventually, for further decreases of $\alpha$, the points of the 2-cycle collide in a saddle point, whose stable manifold separates the basins of attraction of the two Cournot-Nash equilibria, as shown in Figure $12 \mathrm{f}$.

\section{Conclusions}

\section{Acknowledgments}

The work of Fabio Lamantia and Fabio Tramontana is part of the research project Dynamic models for behavioral economics, financed by DESP - University of Urbino, Italy.

\section{Appendix}

\section{Proof of Proposition 1}

Let us first consider a symmetric equilibrium, characterized by the same quantities for both firms $E^{*}=\left(x^{*}, x^{*}\right)$. The equilibrium where both firms innovate, their production must satisfy the equation $x=B_{H}(x)$, i.e. this equilibrium is given by

$$
E_{h h}=\left(\frac{a}{3 b}, \frac{a}{3 b}\right)
$$

The condition for having $E_{h h}$ as an equilibrium is that $\frac{a}{3 b}<\widehat{x}$, i.e., in terms of fixed cost to differentiate that $k<\frac{(a-1)(a+3)}{12 b}$;

Similarly, both players do not innovate and play

$$
E_{l l}=\left(\frac{1}{3 b}, \frac{1}{3 b}\right)
$$

when $\frac{1}{3 b}>\widehat{x}$, i.e. when $k>\frac{(a-1)(3 a+1)}{12 b}$. Notice that when

$$
\frac{(a-1)(a+3)}{12 b}<k<\frac{(a-1)(3 a+1)}{12 b}
$$

neither $E_{h h}$ nor $E_{l l}$ exists (no symmetric equilibrium).

Now we consider asymmetric equilibria. An equilibrium where one firm innovates and chooses $x_{h}^{*}$ whereas the other does not and produces $x_{l}^{*}$ must satisfy the condition:

$$
\left\{\begin{array}{l}
x_{h}^{*}=\frac{a}{2 b}-\frac{x_{l}^{*}}{2} \\
x_{l}^{*}=\frac{1}{2 b}-\frac{x_{h}^{*}}{2}
\end{array}\right.
$$

thus getting the pair of equilibria

$$
E_{h l}=\left(x_{h}^{*}, x_{l}^{*}\right)=\left(\frac{2 a-1}{3 b}, \frac{2-a}{3 b}\right)
$$



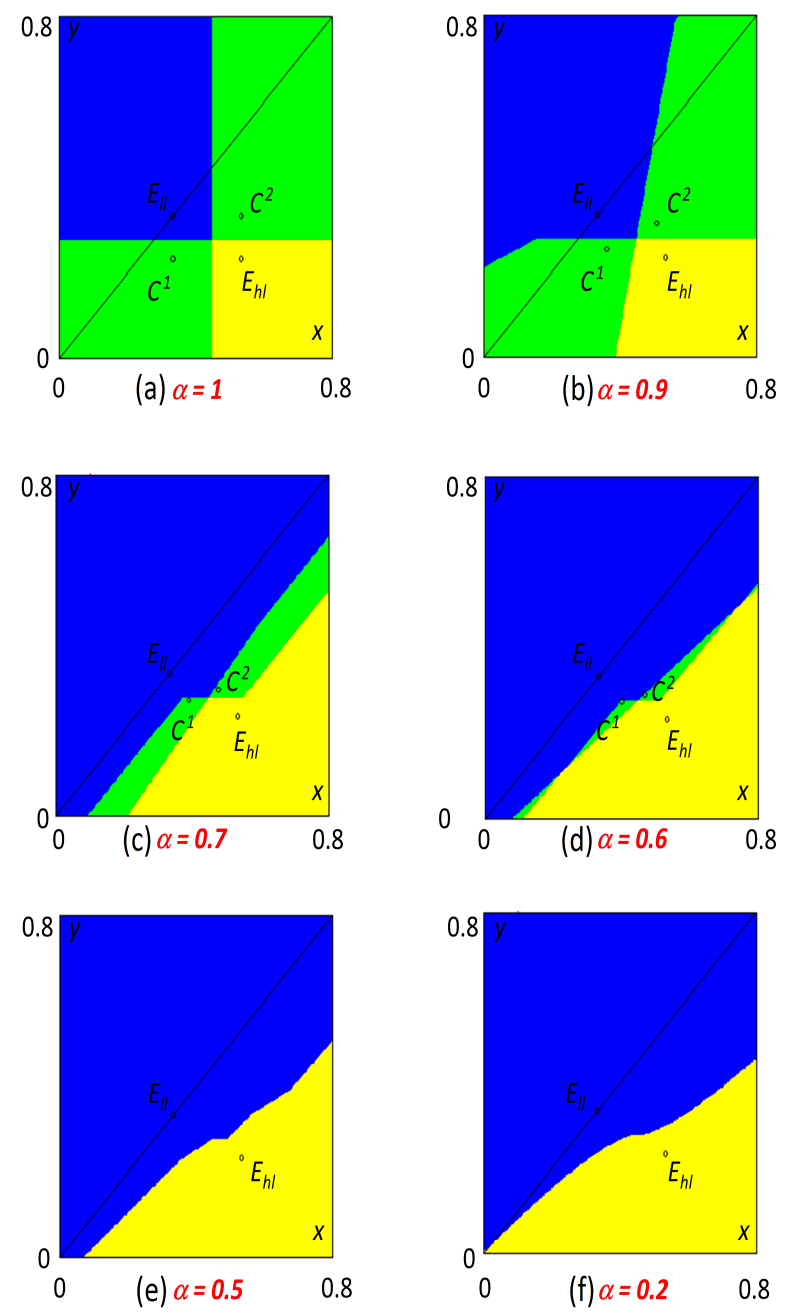

Figure 12: One-sided innovation - Adaptive expectations. Basins of attraction of: $E_{h l}$ (yellow) $E_{l l}$ (blue), and 2-cycle $\left\{C^{1}\left(x_{l}^{*}, f\left(x_{h l}^{*}\right)\right), C^{2}\left(x_{h l}^{*}, x_{l}^{*}\right)\right\}$ (green). Parameters as in Figure 10 for decreasing speeds of adjustment $\alpha$. 
and

$$
E_{l h}=\left(x_{l}^{*}, x_{h}^{*}\right)
$$

Notice that equilibria $E_{h l}$ and $E_{l h}$ are feasible, in the sense that firms find it profitable to play them, provided that $0 \leq x_{l}^{*} \leq \widehat{x} \leq x_{h}^{*}$, i.e. if the following condition holds

$$
\frac{(a-1)(5-a)}{12 b} \leq k \leq \frac{(a-1)(5 a-1)}{12 b}
$$

Moreover, both equilibria $E_{h l}\left(\right.$ and $\left.E_{l h}\right)$ and $E_{l l}$ coexist when:

$$
\frac{(a-1)(1+3 a)}{12 b}<k<\frac{(a-1)(5 a-1)}{12 b}
$$

and that $E_{h l}\left(\right.$ and $\left.E_{l h}\right)$ and $E_{h h}$ coexist when

$$
\frac{(a-1)(5-a)}{12 b}<k<\frac{(a-1)(3+a)}{12 b}
$$

With respect to stability, the Jacobian matrix at equilibrium, be it $E_{h h}, E_{l l}$, $E_{h l}$ or $E_{l h}$, has the form

$$
J(T)=\left(\begin{array}{cc}
1-\alpha & -\frac{\alpha}{2} \\
-\frac{\alpha}{2} & 1-\alpha
\end{array}\right)
$$

for which it is very easy to calculate that its eigenvalues are always in modulus less than one for any $\alpha \in(0,1]$. QED

Proof of Proposition 2

Since best response functions are piecewise-linear, the Jacobian matrix of $T$ (8) $J(T)$ reported in (20) does not depend on the coordinates of the point of the 2-cycle. Being

$$
J^{2}(T)=\left(\begin{array}{cc}
(1-\alpha)^{2}+\frac{\alpha^{2}}{4} & -\alpha(1-\alpha) \\
-\alpha(1-\alpha) & (1-\alpha)^{2}+\frac{\alpha^{2}}{4}
\end{array}\right)
$$

the eigenvalues of $J^{2}, \lambda_{1}=\frac{(\alpha-2)^{2}}{4}$ and $\lambda_{2}=\frac{(3 \alpha-2)^{2}}{4}$, are both in modulus less than one for any $\alpha \in(0,1]$. QED

\section{References}

[1] Bischi, G.I., Chiarella, C., Kopel, M. (2004) The long run outcomes and global dynamics of a duopoly game with misspecified demand functions, International Game Theory Review, Vol. 6, No. 3, 343-379.

[2] Bischi, G.I., Chiarella, C., Kopel, M. and F. Szidarovszky. Nonlinear Oligopolies: Stability and Bifurcations. Springer-Verlag, 2010. 
[3] Bischi G.I. and M. Kopel (2001). "Equilibrium Selection in a Nonlinear Duopoly Game with Adaptive Expectations". Journal of Economic Behavior and Organization. 46 (1), 73-100.

[4] Bischi, G.I., Lamantia F.(2012), Routes to complexity induced by constraints in Cournot oligopoly games with linear reaction functions, Studies in Nonlinear Dynamics \& Econometrics, 16-2.

[5] Bischi G.I., C. Mammana and L. Gardini (2000). "Multistability and cyclic attractors in duopoly games". Chaos, Solitons and Fractals, 11, 543-564.

[6] Cellini, R., Lamantia, F.(2015), Quality Competition in Markets with Regulated Prices and Minimum Quality Standards, Journal of Evolutionary Economics, 25-2, 345-370.

[7] Chiarella, C., He, X.Z. (2003) Dynamics of beliefs and learning under $a_{L^{-}}$ processes - the heterogeneous case, Journal of Economic Dynamics \& Control, 27, 503-531.

[8] Chiarella, C., Szidarovszky F. (2004) Dynamic oligopolies without full information and with continuously distributed time lags, Journal of Economic Behavior \& Organization Vol. 54, 495-511.

[9] Chiarella, C., Szidarovszky F. (2005) Cournot oligopolies with product differentiation under uncertainty, Computers and Mathematics with Applications, 50, 413-424.

[10] Chiarella, C., Szidarovszky F., Zhu P. (2002) The interaction of uncertainty and information lags in the Cournot oligopoly model, in "Oligopoly Dynamics: Models and Tools", T. Puu and I. Sushko (Eds.), Springer Verlag, 233-263.

[11] Dawid, H., Kopel, M., Kort, P.M., (2010). "Innovation threats and strategic responses in oligopoly markets," Journal of Economic Behavior \& Organization, vol. 75(2), 203-222.

[12] Dawid, H., Kopel, M., Kort, P.M., (2013). "New product introduction and capacity investment by incumbents: Effects of size on strategy," European Journal of Operational Research, vol. 230(1), 133-142

[13] Dixit, A.K., Pindyck, R.S., (1994) Investment Under Uncertainty. Princeton University Press, Princeton

[14] Eckert, A., Klumpp, T., Su, X.(2017) An Equilibrium Selection Theory of Monopolization, Southern Economic Journal, DOI: 10.1002/soej.12176

[15] Encaoua, D. and A. Hollander (2002). "Competition Policy and Innovation." Oxford Review of Economic Policy 18, 63-79. 
[16] Evans, D. and R. Schmalensee (2002). "Some Economic Aspects of Antitrust Analysis in Dynamically Competitive Industries" in: A. Jaffe, J. Lerner and S. Stern (eds) innovation Policy and the Economy (Vol 2), 150. Cambridge, MA: MIT Press.

[17] Farrell, J. and M. Katz (2005). "Competition Or Predation? Consumer Coordination, Strategic Pricing and Price Floors in Network Markets." Journal of Industrial Economics 53, 203-231.

[18] Häckner, J., 2000. A Note on Price and Quantity Competition in Differentiated Oligopolies. Journal of Economic Theory 93, 233-239.

[19] Hommes, C.H., (1998). On the consistency of backward-looking expectations: the case of the cobweb. Journal of Economic Behavior and Organizations $33,333-362$.

[20] Marimon, R., Sunder, S., 1993. Indeterminacy of equilibria in a hyperinflationary world: experimental evidence. Econometrica 61, 1073-1107.

[21] Marimon, R., Sunder, S., 1994. Expectations and learning under alternative monetary regimes: an experimental approach. Economic Theory 4, 131162.

[22] Milnor J. (1985) On the concept of attractor. Communications in Mathematical Physics, 99(2):177-195.

[23] Mira C, Gardini L, Barugola A, Cathala JC. Chaotic dynamics in twodimensional noninvertible maps. Singapore: World Scientific; 1996.

[24] Puu, T., Chaos in duopoly pricing, Chaos, Solitons and Fractals, 1, 573, 1991.

[25] Tramontana, F., Gardini L., Puu, T. (2009), "Cournot duopoly when the competitors operate multipleproduction plants", Journal of Economic Dynamics \& Control 33, 250-265

[26] Tramontana, F., Gardini L., Puu, T.(2011), "Mathematical properties of a discontinuous Cournot-Stackelberg model", Chaos, Solitons \& Fractals, 44(1-3), 58-70. 